\title{
Cryo-SEM of Perpendicular Cross Freeze-Fractures Through a High-Pressure-frozen Biofilm
}

\author{
Vladislav Krzyzanek ${ }^{1}$, Kamila Hrubanova ${ }^{1,2}$, Jana Nebesarova $^{3}$ and Filip Ruzicka ${ }^{4}$ \\ 1. Institute of Scientific Instrument ASCR, Department of Electron Microscopy, Brno, Czech Republic \\ 2. Brno University of Technology, Institute of Physical Engineering, Brno, Czech Republic \\ 3. Biology center ASCR, Institute of Parasitology, Ceske Budejovice, Czech Republic \\ 4. Masaryk University, Faculty of Medicine, Brno, Czech Republic
}

The cryo scanning electron microscopy (cryo-SEM) as well as high-pressure-freezing (HPF) belongs to cutting edge techniques in electron microscopy of hydrated samples [1]. However, their combination is not always easily applicable. Here, we present a way of combining high-pressure-freezing using EM PACT2 (Leica Microsystems) that fixes hydrated samples on a $1.4 \mathrm{~mm}$ sapphire discs and high resolution SEM JEOL 7401F equipped with the cryo-attachment ALTO 2500 (GATAN). The freeze-fracturing technique is used here for investigation of yeast cultures cultivated on the sapphire discs, where we focus on the formation of the extracellular matrix produced during the cultivation.

For our experiments we were able to use the HPF instrument EM PACT2 (Leica Microsystems) that freezes samples only on very small sapphire disks with a diameter of $1.4 \mathrm{~mm}$. In order to perform the freeze-fracturing through the small sapphire discs in the cryo-preparation chamber of ALTO 2500, a universal holder was designed and built. There were following requirements for the holder: (1) easy fixing of the sapphire disc in liquid nitrogen, (2) fracturing the sapphire disc with the cultured sample, and (3) possibility to observe both sides of the sapphire disc in the SEM without removing it outside of the ALTO 2500 cryo-attachment. The developed holder is shown in Figure 1a.

For our investigations we used a culture of the yeast Candida parapsilosis that was incubated on the sapphire disks in the YNB medium with $4 \%$ glucose for 20 hours at $37^{\circ} \mathrm{C}$. Frequently researched biofilm-positive Candida parapsilosis strains tested/used in this study were isolated from blood cultures of patients with bacteraemia [1]. The disk was carefully removed from the medium and fixed by the HPF EM PACT2, consequently mounted to the holder in liquid N2 (Figure 1a) and moved into the ALTO 2500 preparation chamber in the standard way. The freeze-fracture technique was applied, i.e. physically breaking the sapphire disc containing the rapidly frozen hydrated sample. In some cases a short freeze-etching was performed and structural details exposed by the fracture plane were then coated by $\mathrm{Pt} / \mathrm{Pd}$. Figures $1 \mathrm{~b}$ and $1 \mathrm{c}$ show fractures of grown cultures of Candida parapsilosis and its extracellular matrix fixed by the two following ways: simple plunging into the liquid ethane (Figure 1b) [3] and use of HPF (Figure 1c). It is obvious that since simple plunging creates ice crystallization, in contrast the HPF preserves the structure well.

Our study of yeast biofilms suggests that cryo-SEM in combination with the high-pressure-freezing and perpendicular cross freeze-fracturing through the sapphire disc is an excellent technique for highly hydrated samples such as biofilms. Thus, details in biofilm formation can be recognized and further studied in their natural hydrated state allowing detailed investigation of ultrafine structure and morphology close to a life-like condition. 


\section{References:}

[1] A Cavalier, D Spehner and BM Humber, Handbook of Cryo-Preparation Methods for Electron Microscopy, (CRC Press, Boca Raton, 2008).

[2] RM Donlan and JW Costerton, Clin. Microbiol. Rev. 15 (2002), p. 167.

[3] K Dobranska et al, Microsc. Microanal. 19 (Suppl 2) (2013), p. 226.

[4] The authors acknowledge the support of grants CZ.1.07/2.3.00/20.0103 and LO1212 (EC and MEYS CR), TE01020118 (TACR) and P205/11/1687 (GACR).

a
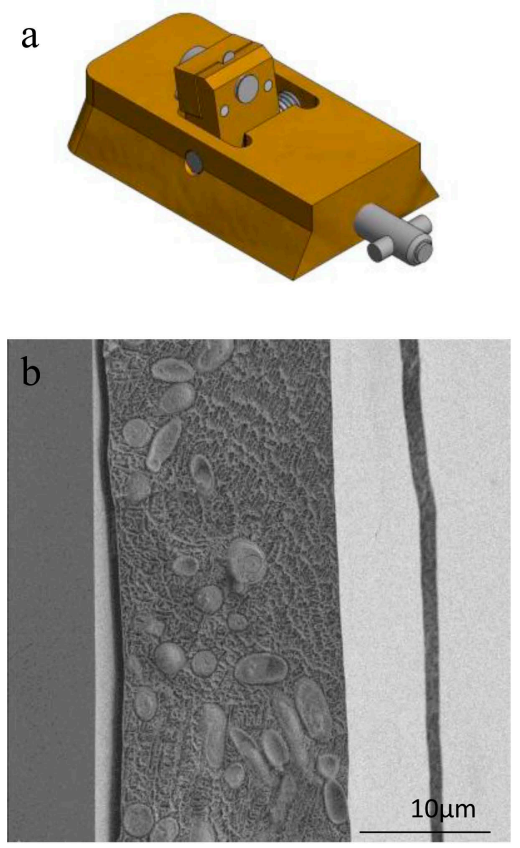

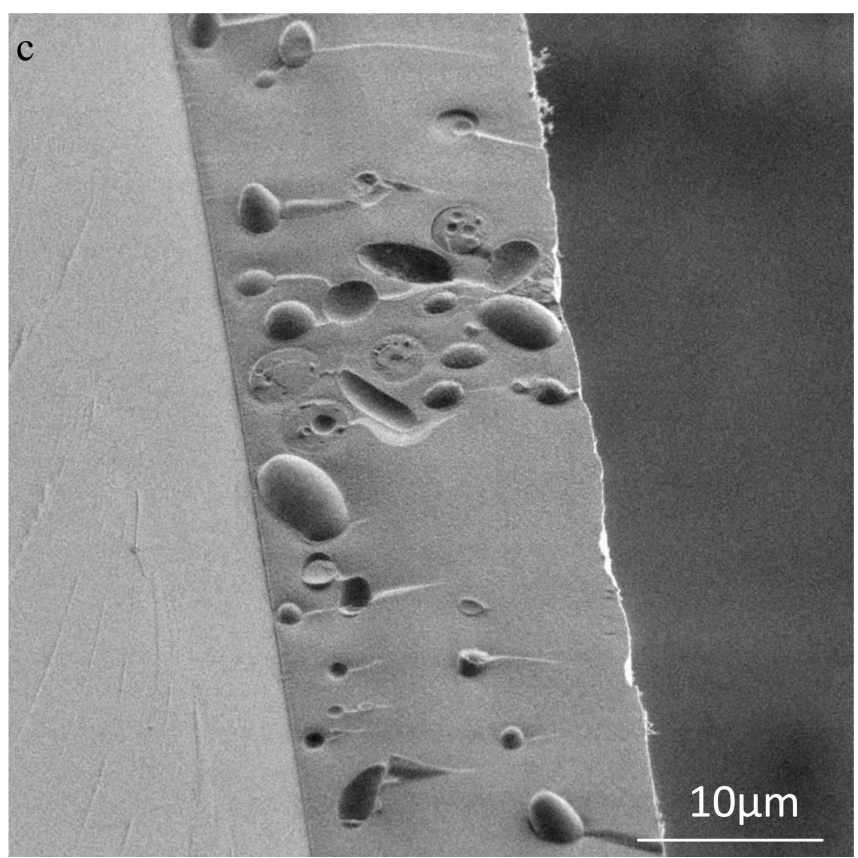

Figure 1. (a) A holder of the $1.4 \mathrm{~mm}$ sapphire disc for the ALTO 2500 cryo-attachment. (b) Freeze-fracture of Candida parapsilosis grown on a glass substrate (left part of the image) and fixed by plunging to the liquid ethane. (c) Freeze-fracture of Candida parapsilosis grown on a sapphire disc (left part of the image) and fixed by HPF. 